\title{
A PHYSICALLY BASED MODEL OF THE IONIZING RADIATION FROM ACTIVE GALAXIES FOR PHOTOIONIZATION MODELING
}

\author{
A. D. Thomas ${ }^{1}$, B. A. Groves ${ }^{1}$, R. S. Sutherland ${ }^{1}$, M. A. Dopita ${ }^{1}$, L. J. Kewley ${ }^{1}$, and C. JiN ${ }^{2}$ \\ ${ }^{1}$ Research School of Astronomy and Astrophysics, Australian National University, Canberra, ACT 2611, Australia; adam.thomas@anu.edu.au \\ ${ }^{2}$ Max-Planck-Institut für Extraterrestrische Physik, Giessenbachstrasse, D-85748 Garching, Germany; chichuan@mpe.mpg.de \\ Received 2016 August 8; revised 2016 October 20; accepted 2016 November 3; published 2016 December 21
}

\begin{abstract}
We present a simplified model of active galactic nucleus (AGN) continuum emission designed for photoionization modeling. The new model OXAF reproduces the diversity of spectral shapes that arise in physically based models. We identify and explain degeneracies in the effects of AGN parameters on model spectral shapes, with a focus on the complete degeneracy between the black hole mass and AGN luminosity. Our reparametrized model OXAF removes these degeneracies and accepts three parameters that directly describe the output spectral shape: the energy of the peak of the accretion disk emission $E_{\text {peak }}$, the photon power-law index of the non-thermal emission $\Gamma$, and the proportion of the total flux that is emitted in the non-thermal component $p_{\mathrm{NT}}$. The parameter $E_{\mathrm{peak}}$ is presented as a function of the black hole mass, AGN luminosity, and "coronal radius" of the OPTXAGNF model upon which OXAF is based. We show that the soft X-ray excess does not significantly affect photoionization modeling predictions of strong emission lines in Seyfert narrow-line regions. Despite its simplicity, OXAF accounts for opacity effects where the accretion disk is ionized because it inherits the "color correction" of OPTXAGNF. We use a grid of MAPPINGS photoionization models with OXAF ionizing spectra to demonstrate how predicted emission-line ratios on standard optical diagnostic diagrams are sensitive to each of the three OXAF parameters. The OXAF code is publicly available in the Astrophysics Source Code Library.
\end{abstract}

Key words: black hole physics - galaxies: individual (NGC 1365) - ISM: lines and bands - line: formation quasars: emission lines

\section{INTRODUCTION}

Active galactic nuclei (AGN) feature narrow-line regions (NLRs) in which continuum radiation from the central engine photoionizes the surrounding interstellar medium (ISM). The relatively hard AGN continuum emission injects more energy and photoionizes the ISM to higher ionization states than the ionizing spectra of $\mathrm{OB}$ stars at the centers of $\mathrm{H}$ II regions.

Many insights into the physics of the the ISM in active galaxies have been gained through the use of photoionization models. The results of photoionization modeling facilitate the routine diagnosis of the density, temperature, and cause of ionization of NLR gas from observed emission-line spectra. Photoionization models have been used to explain the very similar NLR ionization parameters observed across the population of active galaxies - this effect is caused by the gas pressure gradient balancing the radiation pressure gradient in dusty NLR clouds such that the "local" ionization parameter is self-regulated (Dopita et al. 2002; Groves et al. 2004). Models are important tools in investigating the effects of various ISM parameters on emission-line spectra; e.g., determining the effects of changing abundances (Groves et al. 2006). Recently, Davies et al. (2016) showed how comparisons between models and spatially resolved spectroscopy may reveal where extended NLRs are radiation pressuredominated or gas pressure-dominated on scales of tens to hundreds of parsecs.

The emission-line spectra of NLRs in active galaxies strongly depend upon the shape of the spectrum of the central source at energies immediately above the Lyman limit at $13.6 \mathrm{eV}$. These ionizing photons are not directly observable because they are very strongly absorbed in the NLR and broader ISM of the host galaxy, as well as in the ISM of the Milky Way.

Up to the present, ionizing AGN spectra for photoionization modeling have almost exclusively been constructed using piecewise functions of one to three simple power laws (e.g., Viegas-Aldrovandi \& Contini 1989; Allen et al. 1998; Murayama \& Taniguchi 1998; Evans et al. 1999; Contini \& Viegas 2001; Collins et al. 2009). Groves et al. (2004) use a conventional range of power-law indices of $\alpha=-2.0$ to $\alpha=-1.2$, for a single power law covering 0.005 to $1 \mathrm{keV}$. The authors find that a steeper power law leads to weaker highionization lines and relatively stronger $\mathrm{H}$ lines, due to the relative excess of $\mathrm{H}$ - but not He-ionizing photons. A more physically motivated empirical two-component model for the ionizing spectrum is used, for example, in Bland-Hawthorn et al. (2013).

In this work, we seek to develop a modern AGN continuum emission model for use as the input spectrum in photoionization models, based on physically derived models, and including, for example, ionization effects in the shape of the accretion disk spectrum. We aim to identify the important AGN parameters that influence the shape of the ionizing spectrum and hence the predicted emission-line ratios. This allows us to minimize the parameter space used to cover a large range of model spectra. A small parameter space is easier to handle conceptually and computationally, and the process of combining fully and partially degenerate parameters into a smaller set of parameters results in insights into how the shape of the ionizing spectrum may arise from various combinations of fundamental AGN parameters.

In photoionization modeling, we assume that the model ionizing spectrum will be intrinsic when it reaches the 
modeled clouds, i.e., that there is no significant absorption between the central engine and the modeled clouds. Hence, the spectra will primarily feature a peak formed by a superposition of pseudo-blackbodies due to the accretion disk, and a power-law non-thermal component resulting from Compton up-scattering of disk photons. A physical model of these spectra may be expected to be well-described by few parameters.

A detailed model of continuum emission from AGN must rely on theoretical emissivities from accretion disk models. The standard Novikov-Thorne "thin disk" accretion model (Novikov \& Thorne 1973, building on the work of Shakura \& Sunyaev 1973) is applicable over much of the accretion rate regime of Seyfert galaxies $\left(\sim 0.05\right.$ to $\left.\sim 0.3 L / L_{\text {Edd }}\right)$. This model has parameters such as the supermassive black hole (SMBH) mass, spin, and accretion rate, which influence the theoretical ionizing AGN spectrum.

Understanding how the key parameters control the shape of the ionizing EUV-soft X-ray spectrum of AGN allows us to produce a model that keeps the number of free parameters to a minimum. Our simplified spectral modeling is, however, based firmly on the OPTXAGNF model (Done et al. 2012; Jin et al. 2012a, 2012b, 2012c). We chose this model because it is a recent, widely used model, it calculates theoretical spectra using physics such as the Novikov-Thorne thin accretion disk model, and because it uses a "color temperature correction" that accounts for electron-scattering opacity in parts of the disk where hydrogen is ionized. The OPTXAGNF model provides the basis for a new physically based model of the ionizing AGN spectrum for photoionization modeling.

In photoionization modeling, the ionization parameter and density are important input parameters, so the flux incident on the model cloud is easily calculated. However, the actual luminosity of the ionizing source is important only if the modeler is interested in determining the distance of the modeled cloud from the source. We are primarily concerned with the shape of the ionizing spectrum, because this determines the relative emission-line fluxes. Any parameter redundancies may render more difficult the interpretation of the NLR spectrum caused by a given AGN ionizing spectrum. In this work, we identify and explain various spectral-shape degeneracies, such as the complete degeneracy between the black hole mass and luminosity in the Novikov-Thorne emissivity, and successfully remove the degeneracies by constructing a new model.

We simplify the OPTXAGNF model by carefully removing and merging degenerate parameters. The resulting model, OXAF, satisfies our requirements by generating realistic model spectral shapes using the fewest possible parameters, and with all of the parameters having a direct impact on the shape of the model spectrum. We then investigate the effect of the OXAF parameters on photoionization models of NLR clouds.

In Section 2, we describe the spectral-shape degeneracies that arise in the thin disk accretion model and in the the OPTXAGNF model, and in Section 3 we describe the parameters used to reparametrize OPTXAGNF. In Section 4, we explain how these parameters are used to construct our simplified model OXAF and describe the implementation and validation of the new model. In Section 5, we explore how the OXAF parameters affect photoionization model predictions for diagnostic emission lines. Our conclusions are presented in Section 6.

\section{RELATIONSHIPS BETWEEN PARAMETERS IN AGN SPECTRAL MODELS}

The unified model of AGN describes a central, accreting SMBH surrounded by a broad line region, a dusty obscuring torus, and an NLR (Antonucci 1993). The powerful continuum radiation from the central accretion structure illuminates the broad- and narrow-line regions.

Important parameters that determine the spectrum of the central accretion structure are the Eddington ratio $L / L_{\mathrm{Edd}}$, the SMBH mass $M_{\mathrm{BH}}$, the dimensionless SMBH spin $a^{*}$, and the innermost radius at which the disk is directly visible. In this section, we describe the effects of these parameters on the shape of the ionizing spectrum and the relationships between the parameters.

\subsection{The Relationship between the SMBH Mass and Eddington Rate}

The Novikov-Thorne thin disk model produces a clean relationship between $M_{\mathrm{BH}}$ and $L / L_{\mathrm{Edd}}$, with these parameters having the same effect on the model spectral shape.

The temperature $T$ at a given radius of the accretion disk $r_{1}$ may be calculated using the following equation for the "outer" region of the disk (Equation (5.10.1) in Novikov \& Thorne 1973):

$$
T\left(M_{\mathrm{BH}}, \dot{M}_{\mathrm{BH}}, a^{*}, r_{1}\right)=\left[\frac{3 G M_{\mathrm{BH}} \dot{M}_{\mathrm{BH}}}{8 \pi \sigma_{\mathrm{SB}} r_{1}^{3}} \mathcal{B}^{-1} \mathcal{C}^{-1 / 2} \mathcal{Q}\right]^{\frac{1}{4}}
$$

where $M_{\mathrm{BH}}$ is the black hole mass, $\dot{M}_{\mathrm{BH}}$ is the accretion rate, $a^{*}$ is the dimensionless spin parameter, $\sigma_{\mathrm{SB}}$ is the StefanBoltzmann constant, and $G$ is the gravitational constant. With $r$ defined as the radius normalized by the gravitational radius $r_{g}$ $\left(r_{g}=G M_{\mathrm{BH}} / c^{2} ; r=r_{1} / r_{g}\right)$, the quantities $\mathcal{B}\left(r, a^{*}\right), \mathcal{C}\left(r, a^{*}\right)$, and $\mathcal{Q}\left(r, a^{*}\right)$ are complicated dimensionless radial functions associated with relativistic corrections in the analytic accretion disk solution of Novikov \& Thorne (1973; the $\mathcal{Q}$ function was presented in the follow-up work of Page \& Thorne (1974); $\mathcal{B}$, $\mathcal{C}$, and $\mathcal{Q}$ tend to 1 at large $r$ ).

Using $L=\epsilon\left(a^{*}\right) \dot{M}_{\mathrm{BH}} c^{2}$, where $\epsilon\left(a^{*}\right)$ is the total accretion efficiency, and with the definition of $r$ given above, Equation (1) may be rewritten as

$$
T\left(M_{\mathrm{BH}}, L, a^{*}, r\right)=\left[\frac{C}{\epsilon\left(a^{*}\right) r^{3}} \frac{L}{M_{\mathrm{BH}}^{2}} \mathcal{S}\left(r, a^{*}\right)\right]^{\frac{1}{4}},
$$

where $C$ is a collection of physical and numerical constants and $\mathcal{S}\left(r, a^{*}\right)=\mathcal{B}\left(r, a^{*}\right)^{-1} \mathcal{C}\left(r, a^{*}\right)^{-1 / 2} \mathcal{Q}\left(r, a^{*}\right)$.

Inverting Equation (2), we find that

$$
\frac{L / L_{\mathrm{Edd}}}{M_{\mathrm{BH}}}=F\left((r, T), a^{*}\right)
$$

for a function $F$, since $L_{\mathrm{Edd}} \propto M_{\mathrm{BH}}$. Specifying the temperature at a particular normalized radius as well as the spin uniquely determines the ratio $\left(L / L_{\mathrm{Edd}}\right) / M_{\mathrm{BH}}$ such that the parameters $L / L_{\mathrm{Edd}}$ and $M_{\mathrm{BH}}$ are degenerate in their effects on the spectral shape.

\subsection{The OPTXAGNF Model}

The OPTXAGNF model (Done et al. 2012; Jin et al. 2012a, $2012 b, 2012 c)$ is a significant effort in the development of 
theoretical models of continuum radiation from accretion onto black holes that match observed optical, UV, and X-ray continuum spectra. The model is available for use with the XSPEC spectral fitting package, under the name OPTXAGNF.

The model aims to capture the essential features of the continuum emission from a relativistic thin accretion disk with a Comptonizing corona in the equatorial plane of a rotating black hole. It is assumed that material is accreted only through the outer disk, and the released gravitational energy is divided between three spectral components.

1. A pseudo-thermal accretion disk component.

2. A high-energy, power-law non-thermal component, formed by Compton up-scattering from an optically thin, high-temperature medium.

3. An intermediate ("soft X-ray excess") component formed by Compton up-scattering from a Compton-thick, lowtemperature medium.

The model implements the following.

(a) The standard Novikov \& Thorne (1973) thin disk relativistic accretion disk emissivity.

(b) A disk spectrum produced by summing blackbodies in successive annuli, with the temperature of the blackbody spectrum corrected if necessary using an empirical color temperature correction $f_{\text {col }}$. The correction is required due to the absorption opacity varying with temperature, density, and wavelength; in particular, it is used where hydrogen is ionized in the inner parts of the disk in an attempt to account for modifications to the spectrum due to the disk not being locally thermalized and due to electron-scattering opacity (Davis et al. 2006). The correction becomes increasingly important as the temperature of the disk increases (as the luminosity increases or black hole mass decreases).

(c) The disk luminosity between the innermost stable circular orbit (ISCO) $r_{\text {ISCO }}$ and $r_{\text {cor }}$, the "coronal radius," is all emitted as Comptonized radiation.

(d) The Comptonized radiation is split between the soft, intermediate component (assumed to arise in the accretion structure itself) and the hard X-ray power-law tail. The seed photons for Comptonization in both cases come from a blackbody with the same (corrected) temperature as the disk at $r_{\text {cor }}$.

(e) The total luminosity from all three components of the emission (disk, Comptonized soft X-ray excess, Comptonized hard X-ray tail) is fixed by the accretion rate and SMBH spin.

The following are the parameters required by OPTXAGNF to characterize the various physical components of the model.

1. $M_{\mathrm{BH}}$, the black hole mass.

2. $L / L_{\mathrm{Edd}}$, the AGN luminosity in units of the Eddington luminosity, which is proportional to $M_{\mathrm{BH}}$.

3. $a^{*}$, the dimensionless black hole rotation parameter, between 0 and 1 (assuming prograde accretion).

4. $r_{\text {cor }}$, the coronal radius (inner edge of the visible portion of the disk), in units of the gravitational radius $\left(r_{g}=G M_{\mathrm{BH}} / c^{2}\right.$, proportional to $\left.M_{\mathrm{BH}}\right)$.

5. $r_{\text {out }}$, the outer radius of the modeled disk, in units of the gravitational radius, proportional to $M_{\mathrm{BH}}$.

6. $T$, the temperature of the Compton optically thick material that produces the soft X-ray excess.
7. $\tau$, the optical thickness of the Compton optically thick material.

8. $f_{\mathrm{PL}}$, the proportion of the corona power emitted in the hard power-law component; $1-f_{\mathrm{PL}}$ is the proportion in the Compton optically thick component.

9. $\Gamma$, the negative of the photon power-law index of the hard X-ray tail component.

\subsection{Relationships between OPTXAGNF Model Parameters}

We explored the behavior of the disk and non-thermal power-law components of the OPTXAGNF model across a wide parameter space (the parameter ranges given in Section 3.2) and showed that there are strong spectral-shape degeneracies between key parameters. The following sections discuss these degeneracies, which are in addition to the total degeneracy between $M_{\mathrm{BH}}$ and $L / L_{\mathrm{Edd}}$ that originates in the NovikovThorne model.

\subsubsection{Relationship between Disk Coronal Radius, SMBH Mass, and Eddington Rate}

Higher disk temperatures lead to higher-energy disk emission, and in particular the high-energy cutoff of the disk emission is set by the maximum temperature of the directly visible part of the accretion disk. Increasing the temperature of the highest-temperature visible parts of the disk and thereby producing higher-energy disk emission is achievable by increasing $\left(L / L_{\mathrm{Edd}}\right) / M_{\mathrm{BH}}$ (increasing the luminosity and/or decreasing the mass), or alternatively by reducing $r_{\text {cor }}$. Consequently, $r_{\text {cor }}$ is partially degenerate in its effects on the disk spectrum with $L / L_{\mathrm{Edd}}$ and $M_{\mathrm{BH}}$. The degeneracy is not total, because changing the innermost visible normalized radius modifies the shape of the disk spectrum in ways that changing $\left(L / L_{\mathrm{Edd}}\right) / M_{\mathrm{BH}}$ cannot.

\subsubsection{Relationship between SMBH Spin and Disk Coronal Radius}

Changing the spin $a^{*}$ with the other parameters fixed has the effect of changing the relative flux contribution of the disk and non-thermal components. This effect is illustrated in Figure 1, which shows models with no intermediate component. As the spin of an SMBH increases, more energy is released by infalling matter; the additional energy is released close to the SMBH event horizon. When we fix the mass, coronal radius, and luminosity, increasing the spin serves primarily to apportion more of the fixed luminosity to the part of the disk within the coronal radius, and hence to the non-thermal component. Hence $a^{*}$ has a similar effect to $r_{\text {cor }}$ in that it changes the relative contributions of the disk and non-thermal components.

Figure 1 shows that the shape of the two spectral components is not strongly affected by $a^{*}$ (with $r_{\text {cor }}=10 r_{g}$ ). However, the parameters $r_{\text {cor }}$ and $a^{*}$ are not entirely degenerate in their effects on the spectral shape, since $a^{*}$ affects the spectral shape through modifying the radial functions $\mathcal{B}\left(r, a^{*}\right)$, $\mathcal{C}\left(r, a^{*}\right)$, and $\mathcal{Q}\left(r, a^{*}\right)$ in Equation (1), whereas $r_{\text {cor }}$ determines which values of $r$ contribute to the disk emission.

The full and partial degeneracies discussed in the previous sections suggest that $M_{\mathrm{BH}}, L / L_{\mathrm{Edd}}, r_{\mathrm{cor}}$, and $a^{*}$ could be combined into two parameters in a simplified model of the spectral shape-one parameter that shifts the disk emission in 


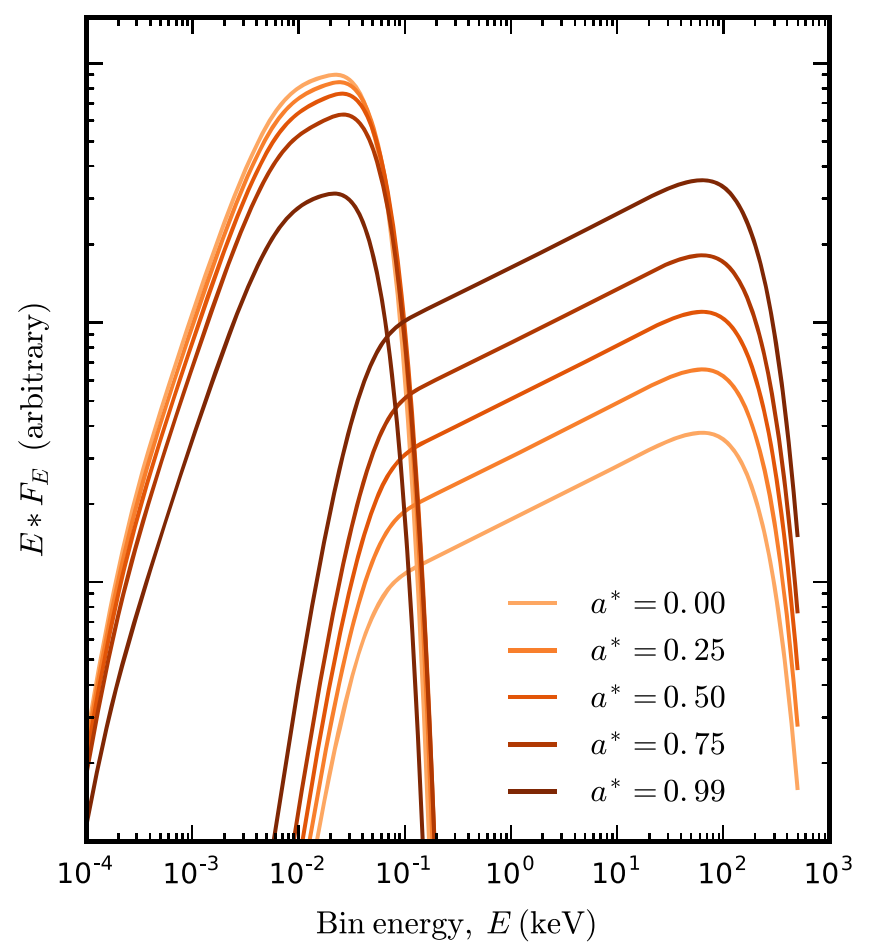

Figure 1. Disk and high-energy non-thermal emission components for varying spin parameter $a^{*}$, with the other key OPTXAGNF parameters fixed at $M_{\mathrm{BH}}=10^{8} M_{\odot}, L / L_{\mathrm{Edd}}=0.1, r_{\mathrm{cor}}=10 r_{g}$, and $\Gamma=1.8$. The intermediate component is not considered $\left(f_{\mathrm{PL}}=1\right)$. Varying $a^{*}$ changes the relative contributions of the disk and non-thermal components to the total spectrum. Note that the shape of these components remains relatively independent of $a^{*}$.

energy space and one parameter that controls the relative flux between the disk and non-thermal components.

\section{SHRINKING THE SPECTRAL MODEL PARAMETER SPACE}

We reduce the free parameter space of the OPTXAGNF model by fixing or neglecting some parameters and by reparametrizing the remaining parameter space.

\subsection{Neglected or Fixed Parameters}

\subsubsection{Intermediate Soft X-Ray Component}

The origin of the widely observed soft X-ray excess in AGN remains uncertain, despite recent progress. In particular, two general explanations are currently favored that attempt to explain the relatively constant energy of the soft excess $\left(k T_{e} \sim 0.1-0.2 \mathrm{keV}\right)$. These explanations are Comptonized disk emission (e.g., the treatment in OPTXAGNF), and blurred reflection from the ionized accretion disk (e.g., Walton et al. 2013). Additionally, absorption has been seriously considered (e.g., Gierliński \& Done 2004), and there is evidence that a contribution to the soft X-ray excess comes from the wider NLR (e.g., Bianchi et al. 2006).

The possible physical processes that give rise to the centrally originating soft excess may be constrained using hard and soft time lags (hard X-rays lagging soft X-rays and soft X-rays lagging hard X-rays) and correlations between spectral components. The time-delay of soft X-ray time lags is strongly correlated with black hole mass (De Marco et al. 2013).
Each soft X-ray excess photon with energy in the range of $\sim 0.2-2 \mathrm{keV}$ will have less effect on the ionization state of the nebula than a photon with energy in the range of 0.01 $-\sim 0.2 \mathrm{keV}$. In the pilot study of Dopita et al. (2014), we found that the intermediate soft X-ray component was not required to achieve satisfactory fits to the optical emission-line spectrum of the Seyfert galaxy NGC 5427 with photoionization models; though, the observed spectrum did not include highionization lines. We do, however, expect that the abundances of high-ionization potential species such as Ar X (423 eV), Fe XIV $(361 \mathrm{eV})$, and Fe X $(234 \mathrm{eV})$ would be affected by dominant soft X-ray excesses. Additionally, a strong soft X-ray excess extends the partially ionized zone, where Auger electrons produced by X-ray bombardment partially ionize the gas, and therefore must, to some extent, enhance emission of lines such as [O I] and [S II].

We performed an investigation on the effect of the soft excess on photoionization modeling of NLR clouds, using ionizing spectra from the OPTXAGNF model. The parameters of the MAPPINGS ${ }^{3} 5.1$ (R. S. Sutherland et al. 2017, in preparation) models and OPTXANGF spectra were fixed, apart from two quantities: the relative strength of the soft excess, which ranged from $0 \%$ to $100 \%$ of the non-thermal flux, and the coronal radius, which ranged from $r_{\text {cor }}=20$ to $r_{\text {cor }}=60$. The models were ionization-bounded and ended when the nebula was $99 \%$ neutral. The results are presented in standard optical diagnostic diagrams (Baldwin et al. 1981; Veilleux \& Osterbrock 1987) in Figure 2, with the fiducial model parameter values listed in the caption. The standard optical diagnostic diagrams use ratios of lines associated with different ionization levels in the gas to distinguish nebulae based on the cause of ionization. Ionization increases from bottom-left to top-right on the diagrams. The lower-left of each of the diagnostic diagrams is associated with $\mathrm{H}$ II regions of varying metallicity and ionization parameter, whereas the top-right is associated with ionization by harder AGN spectra or shocks. The standard dividing lines on the diagrams are described in the caption.

The results shown in Figure 2 confirm that the soft X-ray excess is not of primary importance when predicting diagnostic optical emission-line ratios. The line ratios are evidently most sensitive to variation in the coronal radius or weighting of spectral components when most of the energy released inside the coronal radius is emitted in the power-law component as opposed to the soft excess. For the tested $r_{\text {cor values larger than }}$ $20 r_{g}$, the $[\mathrm{S} \mathrm{II}] / \mathrm{H} \alpha$ and $[\mathrm{OI}] / \mathrm{H} \alpha$ ratios are significantly increased by increasing the proportion of the flux emitted in the power-law tail, resulting in some outlying points to the right of the second and third panel in the figure. The positions of these points are due to the partially ionized zone being extended by hard X-rays in improbably hard ionizing spectra. If we do not consider these power-law dominated models (shown for completeness), the range of points representing the different soft excess strengths is small compared to the variation that may be produced by varying the ionization parameter or metallicity, for example. If the proportion of the energy emitted in the soft excess as opposed to the power-law component does not vary as strongly between real objects as it does in our experiment, and additionally if real objects are mass-bounded as opposed to ionization-bounded (i.e., hard X-rays escape the

\footnotetext{
Available at http://miocene.anu.edu.au/Mappings.
} 


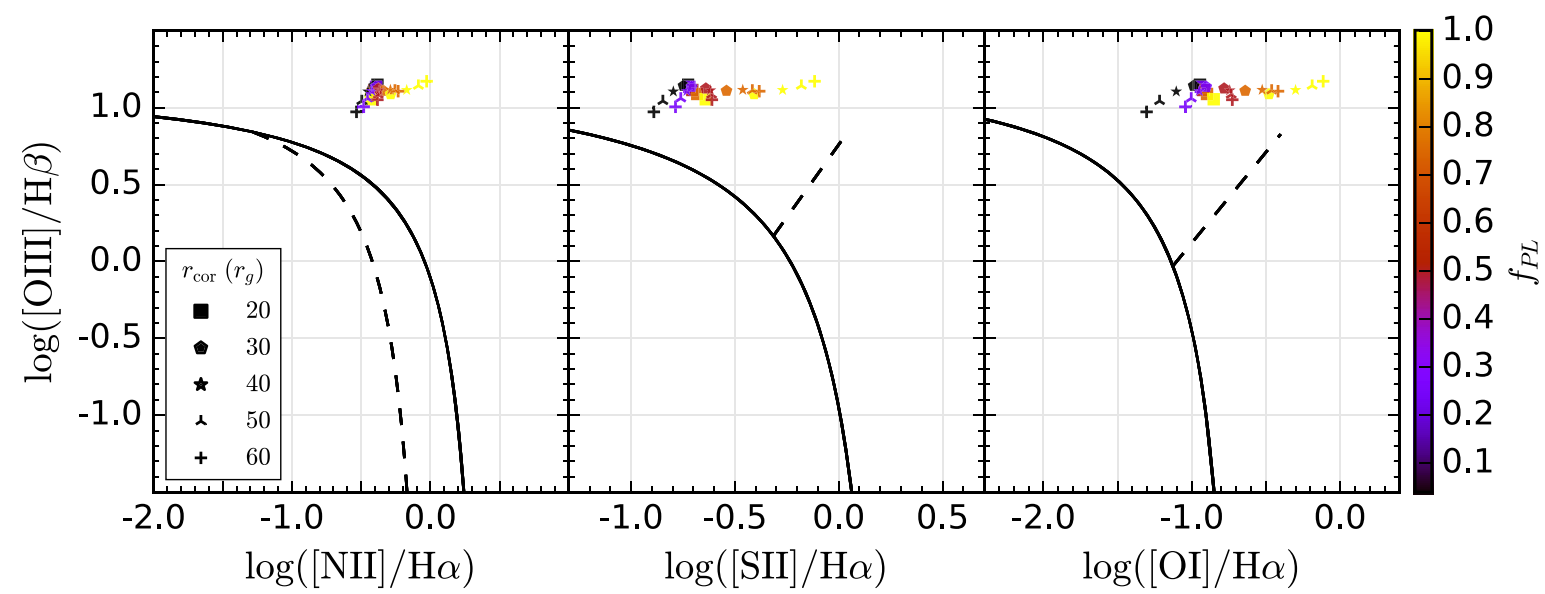

Figure 2. Predicted emission-line ratios produced by MAPPINGS photoionization models using OPTXAGNF input ionizing spectra, shown on optical diagnostic diagrams (Baldwin et al. 1981; Veilleux \& Osterbrock 1987). The solid lines (Kewley et al. 2001) and the dashed line in the left panel (Kauffmann et al. 2003) divide $\mathrm{H}$ II regions (below) and nebulae with higher ionization states (above). The dashed lines in the rightmost panels (Kewley et al. 2006) divide LINERs (below) and Seyferts (above). The fraction of the energy inside the coronal radius that goes to the power-law component as opposed to the intermediate Comptonized component was set to each of $f_{\mathrm{PL}}=0.04,0.25,0.5,0.75$, and 1.00, and for each of these values we considered coronal radii of $r_{\mathrm{cor}}=20,30,40,50$, and $60 r_{g}$. Points are colored by $f_{\mathrm{PL}}$ and the marker shape indicates $r_{\mathrm{cor}}$. We argue in Section 3.1.1 that the results in this figure demonstrate that photoionization modeling of the strong optical lines is insensitive to the soft X-ray excess. For this experiment, the MAPPINGS models were configured with metallicity $Z=2 Z_{\odot}$, ionization parameter $U(H)=10^{-2}$, and a constant pressure of $P / k=10^{6} \mathrm{~K} \mathrm{~cm}^{-3}$. The other parameters of OPTXAGNF were set to $M_{\mathrm{BH}}=5 \times 10^{7} M_{\odot}, L / L_{\mathrm{Edd}}=10^{-0.5}, a^{*}=0, \Gamma=2.0$, and (for the Comptonized soft excess) $k T=0.2 \mathrm{keV}$ with $\tau=15$.

NLR), then the ranges shown in Figure 2 would represent approximate upper bounds on the sensitivity to the soft excess.

In the remainder of this work, we do not include the intermediate Comptonized component, such that the fraction of the corona power in the power-law tail is set to $f_{\mathrm{PL}}=1$, and the parameters for the temperature and optical thickness of the Comptonizing optically thick medium have no effect.

Neglecting the intermediate component enables a vast simplification of the model. Including the soft excess would complicate the comparison of photoionization models to observed NLR emission-line spectra due to uncertainty regarding the origin, time-averaged behavior, and importance relative to other spectral components of the soft X-ray excess. We note that recently Pal et al. (2016) attributed $\sim 60 \%-80 \%$ of the $\sim 0.3-2 \mathrm{keV}$ emission observed in the AGN II Zw 177 to the soft excess, with a significant variation observed between observations separated by 11 years. Another recent work is a study of the AGN RE J1034+396 (Czerny et al. 2016), in which the best-fitting models suggested that $\sim 90 \%$ of the power produced inside the coronal radius is emitted in the intermediate component. Although the strength of the soft excess has been measured in sources such as II Zw 177 and RE J1034+396, the properties of the soft X-ray excess across the population of Seyfert galaxies, not just those that have been studied in X-rays, are unknown.

\subsubsection{The Black Hole Spin}

The spins of astrophysical black holes have long been expected to be non-zero. It is now routine to "map" the central $\sim 20 r_{g}$ of AGN accretion structures by studying X-ray reverberation lags and blurred accretion disk reflection (Fabian 2016). Identifying the innermost radius required by spectral blurring analysis with the ISCO allows the black hole spin to be measured. Spin measurements performed to date generally demonstrate high spins (Reynolds 2014). However, because radiative efficiency increases by up to a factor of five with increasing spin, the very high calculated spins for many AGN with spin measurements are associated with a strong selection bias (Fabian 2016).

As demonstrated above in Section 2.3, the black hole spin is partially degenerate with $r_{\text {cor }}$ in that it changes the relative contributions of the disk and non-thermal components to the total spectrum. The coronal radius will be set larger than radii for which we expect the disk spectrum shape to be strongly affected by the SMBH spin. Figure 1 shows that changing the spin changes the relative energy in the disk and non-thermal components by an order of magnitude (between spins of zero and one); in our simplified model, this effect will be accounted for by an explicit parameter for this scaling. The SMBH spin was set to $a^{*}=0$.

\subsubsection{Outer Edge of Accretion Disk}

The outer disk produces non-ionizing optical and IR emission, and therefore does not contribute to the NLR heating. The parameter defining the outer edge of the accretion disk was set to $10^{4} r_{g}$, a default value.

\subsection{Reparametrizing the AGN Continuum Model}

As a result of the simplifications discussed in the Section 3.1, the OPTXAGNF parameters have been reduced to the four most important parameters $M_{\mathrm{BH}}, L, r_{\mathrm{cor}}$, and $\Gamma$. Exploration of the properties of the theoretical AGN spectra and development of a simplified model (Section 4) were performed over the following ranges of these four parameters.
(a) $-5.0 \leqslant \log _{10}\left(L / L_{\text {Edd }}\right) \leqslant 0.0$
(b) $6.0 \leqslant \log _{10}\left(M_{\mathrm{BH}} / M_{\odot}\right) \leqslant 9.0$
(c) $1.4 \leqslant \Gamma \leqslant 2.6$
(d) $10 r_{g} \leqslant r_{\text {cor }} \leqslant 100 r_{g}$

The maximum luminosity of Eddington was chosen because the Novikov-Thorne thin disk model cannot plausibly be used 
beyond this limit; indeed a slim disk prescription is necessary for a luminosity above $\sim 0.3 L / L_{\text {Edd }}$. The range of $\mathrm{SMBH}$ masses covers the observed range in AGN except for extreme objects (see Figure 4 in Vika et al. 2009). The broad range in $\Gamma$ covers the approximate range of observed values (see Figure 4 in Molina et al. 2013), corresponding to very soft $(\Gamma=2.6)$ through to very hard $(\Gamma=1.4)$ power-law tails.

The physical extent of the corona has been constrained using an analysis of blurred X-ray reflection, modeling of X-ray reverberation, and microlensing observations; these methods suggest that the Comptonizing corona lies within approximately $10 r_{g}$ of the black hole (Fabian 2016). However, because of the selection bias discussed in Section 3.1.2, coronal geometries inferred in X-ray studies are not necessarily representative of the population of Seyfert galaxies. We chose a lower bound for the coronal radius of $10 r_{g}$ here; our experimentation showed that below this value the high-energy part of the accretion disk emission becomes too strongly affected by relativistic corrections for easy simplification of the model. Setting a minimum coronal radius of $10 r_{g}$ will primarily affect the high-energy part of the accretion disk emission by removing it from the disk spectrum. The top end of the $r_{\text {cor }}$ range was selected to include the range suggested by Done et al. (2012).

\subsubsection{Reparametrizing the Disk Component}

A highly desirable simplification is combining the three fully or partially degenerate parameters $L, M_{\mathrm{BH}}$, and $r_{\text {cor }}$ into a single parameter that is able to parametrize (at least approximately) the position in energy space and shape of the Big Blue Bump (BBB) disk emission. The parameter that was chosen to replace the degenerate trio $\left(L, M_{\mathrm{BH}}, r_{\text {cor }}\right)$ was $E_{\text {peak }}$, the peak of the $\mathrm{BBB}$ when the disk spectrum is given in a log-log plot of energy flux versus energy (e.g., Figure 1). The location of the single peak is a reliable and easily calculated feature of a model BBB.

The value of $E_{\text {peak }}$ was determined for a grid of OPTXAGNF spectra over the relevant ranges of the three parameters affecting $E_{\text {peak }}$, which are $L, M_{\mathrm{BH}}$, and $r_{\text {cor }}$. The results were systematically analyzed for each value of $r_{\text {cor }}$, before all of the analyses for various $r_{\text {cor }}$ values were combined into an empirical formula for calculating $E_{\text {peak }}$ as a function of $(L$, $\left.M_{\mathrm{BH}}, r_{\mathrm{cor}}\right)$. An example of the analysis that was applied for a single value of $r_{\text {cor }}$ is presented in Figure 3.

Figure 3 demonstrates how fits for $E_{\text {peak }}$ at a given $r_{\text {cor }}$ must account for the "piecewise" behavior of $E_{\text {peak }}$ as a function of $M_{\mathrm{BH}}$ and $L$. Our solution to this problem was to use a combination of linear and cubic functions as described in the figure and caption. The coefficients of these linear and cubic functions varied as a function of $r_{\text {cor }}$. To construct a general function applicable over the entire three-dimensional $\left(L, M_{\mathrm{BH}}\right.$, $r_{\text {cor }}$ ) parameter space, we required a means to determine the coefficients of the linear and cubic functions from $r_{\text {cor }}$. Linear and quadratic functions of $\log r_{\text {cor }}$ produced satisfactory fits to the values of these coefficients.

The analysis in Figure 3 was applied for $r_{\text {cor }}=6,10,15,24$, 39,63 , and $100 r_{g}$. The analysis for $r_{\text {cor }}=6 r_{g}$ (i.e., at the ISCO, such that the corona is effectively absent) was found to be generally inconsistent with the analyses for the larger $r_{\text {cor }}$ values, presumably due to the effect of strong general relativistic corrections. We thus did not use the analysis for $r_{\text {cor }}=6 r_{g}$.

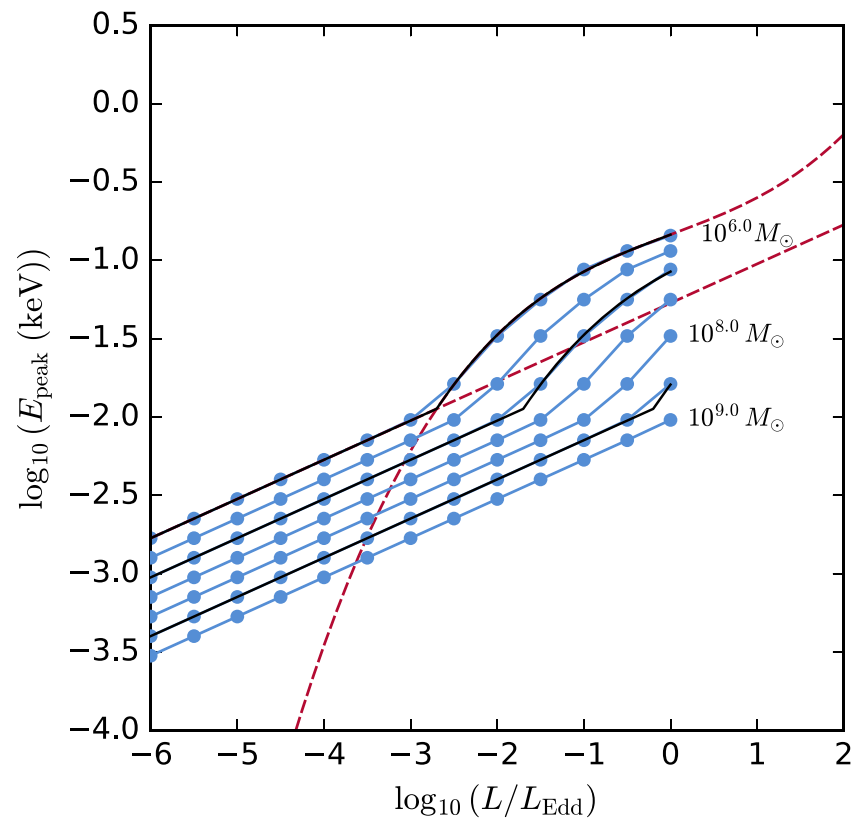

Figure 3. We can characterize the dominant AGN emission in the EUV, the Big Blue Bump (BBB), in terms of the peak of its energy distribution, $E_{\text {peak }}$. Here we show the $E_{\text {peak }}$ predicted by OPTXAGNF as a function of $L / L_{\text {Edd }}$ (blue points) and $M_{\mathrm{BH}}$ (blue curves as labeled, spaced at $0.5 \mathrm{dex}$ ), for $r_{\mathrm{cor}}=24.5 r_{\mathrm{g}}$. To predict $E_{\text {peak }}$ for a given $r_{\text {cor }}$ and $M_{\mathrm{BH}}$, we use a model with the functional form $\max \left(f_{1}\left(L / L_{\mathrm{Edd}}\right), f_{2}\left(L / L_{\mathrm{Edd}}\right)\right)$ (Equation (4)) where $f_{1}$ is a power law (at low $L / L_{\text {Edd }}$ ) and $f_{2}$ is a $\log$-space cubic (at high $L / L_{\text {Edd }}$ ). Both $f_{1}$ and $f_{2}$ are illustrated for $M_{\mathrm{BH}}=10^{6}$ by the red dashed curves. Model fits for $M_{\mathrm{BH}}=10^{6.0}, 10^{7.0}$ and $10^{8.5} M_{\odot}$ are overlaid in black.

Table 1

Fit Parameters for Equations (5) and (6)

\begin{tabular}{lccc}
\hline \hline Parameter & Value & Parameter & Value \\
\hline$A_{x}^{2}$ & $-0.18 \pm 0.04$ & $b_{1, x^{2}}$ & $0.391 \pm 0.008$ \\
$A_{x}^{1}$ & $0.0 \pm 0.1$ & $b_{1, x^{1}}$ & $-0.83 \pm 0.02$ \\
$A_{x}^{0}$ & $0.59 \pm 0.08$ & $b_{1, x^{0}}$ & $0.61 \pm 0.02$ \\
$B$ & $0.250 \pm 0.004$ & $a_{x}^{2}$ & $-0.30 \pm 0.03$ \\
$b_{3, x^{1}}$ & $0.034 \pm 0.002$ & $a_{x}^{1}$ & $0.4 \pm 0.1$ \\
$b_{3, x^{0}}$ & $-0.019 \pm 0.003$ & $a_{x}^{0}$ & $-0.82 \pm 0.07$ \\
\hline
\end{tabular}

The combined analysis for the various $r_{\text {cor }}$ values culminated in the following equation for a hypersurface to predict $E_{\text {peak }}$ as a function of $L, M_{\mathrm{BH}}$, and $r_{\mathrm{cor}}$,

$$
\log _{10} E_{\text {peak }}\left(L, M_{\mathrm{BH}}, r_{\text {cor }}\right)=\max \left(f_{1}, f_{2}\right) \text {, }
$$

where

$$
\begin{aligned}
f_{1}\left(L, M_{\mathrm{BH}}, r_{\mathrm{cor}}\right)= & \left(A_{x^{2}} R\left(r_{\mathrm{cor}}\right)^{2}+A_{x^{1}} R\left(r_{\mathrm{cor}}\right)+A_{x^{0}}\right) \\
& +B S\left(L, M_{\mathrm{BH}}\right)
\end{aligned}
$$

and

$$
\begin{aligned}
f_{2}(L, & \left.M_{\mathrm{BH}}, r_{\mathrm{cor}}\right) \\
= & \left(b_{3, x^{1}} R\left(r_{\mathrm{cor}}\right)+b_{3, x^{0}}\right)\left(S\left(L, M_{\mathrm{BH}}\right)+6\right)^{3} \\
& +\left(b_{1, x^{2}} R\left(r_{\mathrm{cor}}\right)^{2}+b_{1, x^{1}} R\left(r_{\mathrm{cor}}\right)+b_{1, x^{0}}\right)\left(S\left(L, M_{\mathrm{BH}}\right)+6\right) \\
& +\left(a_{x^{2}} R\left(r_{\mathrm{cor}}\right)^{2}+a_{x^{1}} R\left(r_{\mathrm{cor}}\right)+a_{x^{0}}\right) .
\end{aligned}
$$


Here the energy of the $\mathrm{BBB}$ peak $E_{\text {peak }}$ is in $\mathrm{keV}$, $S\left(L, M_{\mathrm{BH}}\right)=\log _{10}\left(\left(L / L_{\mathrm{Edd}}\right) / M_{\mathrm{BH}}\right)$, where $M_{\mathrm{BH}}$ is in $M_{\odot}$, and $R\left(r_{\text {cor }}\right)=\log _{10}\left(r_{\text {cor }} / r_{g}\right)$. The fit parameters are given in Table 1. Note that $L$ and $M_{\mathrm{BH}}$ appear explicitly in Equations (5) and (6) only through the quantity $S\left(L, M_{\mathrm{BH}}\right)$, due to the complete degeneracy in their effects on the spectral shape (Section 2.1; note that there is an implicit dependence on $M_{\mathrm{BH}}$ in the normalization of $L$ and $r_{\text {cor }}$ ). The arbitrary constant 6 occurs with $S\left(L, M_{\mathrm{BH}}\right)$ in Equation (6) only because it was propagated through the analysis from the cubic functions such as those in Figure 3, where the cubic was fitted to the $\log M_{\mathrm{BH}} / M_{\odot}=6$ data (the lowest $M_{\mathrm{BH}}$ value we considered), and was shifted appropriately to apply to other $M_{\mathrm{BH}}$ values. Equations (4) to (6) in conjunction with Table 1 predict $E_{\text {peak }}$ with a standard deviation of $\sim 2 \%$ in linear energy space.

The shape of the BBB spectrum depends (to first order) only on the energies it covers, so we use $E_{\text {peak }}$ to parametrize both the location in energy space and shape of the BBB emission. The construction of a simplified BBB model using this approach is discussed in Section 4.1.

\subsubsection{Reparametrizing the Non-thermal Component}

The shape of the non-thermal component is determined by the shape of the seed photon spectrum and by the parameter $\Gamma$. The seed photon spectrum is a blackbody with a temperature that will be determined by the single parameter $E_{\text {peak }}$, so we have already reparametrized the shape of the non-thermal component such that it depends on only two parameters. The construction of the non-thermal emission component is discussed in Section 4.2.

\section{THE OXAF MODEL}

This section describes the construction of the OXAF model. In particular, OXAF is a three-parameter model, and the name OXAF, a contraction of OPTXAGNF, was chosen to reflect that OXAF is in some sense a "reduced" OPTXAGNF.

The three parameters of the OXAF model are the energy of the peak of the BBB $E_{\text {peak }}$, the photon power-law index $\Gamma$, and the proportion of the total energy flux emitted in the nonthermal component $p_{\mathrm{NT}}$, with the remainder being in the $\mathrm{BBB}$.

In OPTXAGNF, the shape of the BBB and the proportion of energy that goes to the power-law component are not independent, because both are affected by $r_{\text {cor }}$. However, in OXAF, the parameters $E_{\text {peak }}$ (which determines the BBB shape) and $p_{\mathrm{NT}}$ are independent by construction. For a given $E_{\text {peak }}$, OXAF does not have any parameters available to reproduce the variation in OPTXAGNF BBB shapes that occurs as $r_{\text {cor }}$ changes. However, the variation of the $\mathrm{BBB}$ shape with $r_{\text {cor }}$ is a small effect, so a single OXAF BBB is able to satisfactorily reproduce OPTXAGNF BBBs, which have the same peak energy but a range of $r_{\text {cor }}$ (Section 4.4).

The following sections show how we construct a BBB using only one parameter, the energy of the $\mathrm{BBB}$ peak $E_{\text {peak }}$, and a non-thermal component using only two parameters $\left(E_{\text {peak }}\right.$ and $\Gamma$ ).

\subsection{Modeling the Accretion Disk Emission}

The $\mathrm{BBB}$ is parametrized using only one parameter, the energy of the BBB peak $E_{\text {peak }}$. The OXAF model must use this

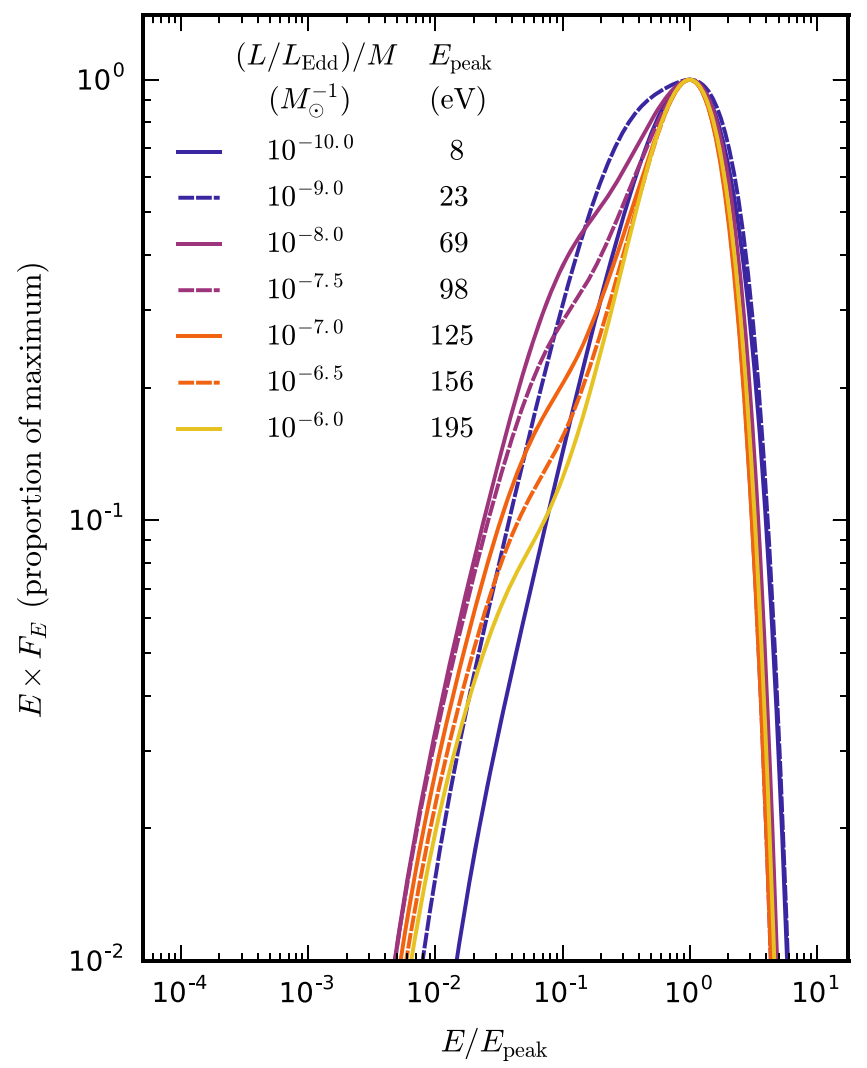

Figure 4. Normalized Big Blue Bump (BBB) accretion disk component OPTXAGNF spectra, shown for varying $\left(L / L_{\mathrm{Edd}}\right) / M_{\mathrm{BH}}$ and for $r_{\mathrm{cor}}=10 r_{g}$. The models were generated using a standalone version of OPTXAGNF produced using a simple fortran wrapper. The shape of the BBB changes due to the increasingly important color correction for higher-energy BBBs. The curves are normalized to have the same flux in the bin with maximum flux, and shifted in logarithmic energy space to have coinciding peaks.

input to determine the shape of the $\mathrm{BBB}$, in particular, for relatively high $\mathrm{BBB}$ peak energies, when the color correction becomes important.

The modeled BBBs are considered in terms of log-log plots of energy flux (i.e., $\mathrm{keV} \mathrm{cm}^{-2} \mathrm{~s}^{-1}$ ) versus energy. The effects of the color correction in this space are illustrated in Figure 4.

The OXAF model constructs the BBB as follows: sixth-order polynomials were fit to a series of OPTXANGF BBBs at different energies $\left(\log _{10}\left(E_{\text {peak }} / \mathrm{keV}\right)=-3.0,-2.4,-1.9,-1.4,-1.2\right.$, $-0.7)$ roughly representative of the range of shapes shown in Figure 4. The BBBs were normalized to have the same peak flux and position of the peak flux in energy space before fitting. The coefficients of the fits are stored in the OXAF code. To determine the shape of an OXAF BBB for a given input $E_{\text {peak }}$, the code (log-)linearly interpolates between the polynomial fits for the nearest $E_{\text {peak }}$ values above and below the input $E_{\text {peak }}$. The resulting polynomial BBB shape is a weighted sum of the two "nearest" polynomials. The interpolated polynomial is shifted in energy space so the peak aligns with the input $E_{\text {peak }}$ value.

\subsection{Modeling the Non-thermal Emission}

The non-thermal component is parametrized using two quantities: $E_{\text {peak }}$ and $\Gamma$. 
The OXAF model uses the Nthcomp routine to generate an inverse-Compton scattered non-thermal component. The fortran subroutines donthcomp (Zdziarski et al. 1996; Życki et al. 1999) and thcompton with thermlc (Lightman \& Zdziarski 1987) from XSPEC were incorporated into OXAF.

There is one difference in the modeling of the non-thermal component between OXAF and OPTXAGNF. In OPTXAGNF, the seed blackbody spectrum temperature is set to $T_{\text {cor }}$, the colorcorrected temperature of the disk at $r_{\text {cor }}$. To approximate this method in OXAF it was necessary to convert the $E_{\text {peak }}$ parameter to an estimate of $T_{\text {cor }}$, despite the OXAF model lacking an explicitly calculated $r_{\text {cor }}$. The conversion was achieved by extracting $T_{\text {cor }}$ values for 72 representative OPTXAGNF models across the parameter space and relating them to the $E_{\text {peak }}$ values calculated for these models using Equation (4). The following linear fit from this comparison is used by OXAF to relate the two parameters,

$$
\log _{10}\left(k T_{\text {cor }}\right)=0.96 \log _{10}\left(E_{\text {peak }}\right)-0.39
$$

where both $k T_{\text {cor }}$ and $E_{\text {peak }}$ are measured in $\mathrm{keV}$. Here the gradient and intercept have errors of 0.02 and 0.03 dex respectively, and the resulting error on $\log _{10}\left(k T_{\text {cor }}\right)$ is 0.07 dex. The non-thermal spectrum is constructed by converting $E_{\text {peak }}$ to $T_{\text {cor }}$ using Equation (7) before inputting $\Gamma, T_{\text {cor }}$, and an assumed Comptonizing plasma temperature of $100 \mathrm{keV}$ into the OXAF donthcomp function, which in turn calls thcompton, which calls thermlc.

\subsection{Implementation}

The OXAF model was implemented as a module oxaf.py written in the programming language python, with a design focus on convenience of use. The module depends only on the standard third-party numerical Python library numpy, it is a single file that may be used with both python 2 and python 3 and it requires no compilation step to run (the included fortran codes were converted to python). The module may simply be run as a command line script to output a model spectrum to stdout, or alternatively may be imported to be used in python code. The module is thoroughly self-documented and is included in the Astrophysics Source Code Library (ASCL). ${ }^{4}$

The oxaf.py module includes functions to find $E_{\text {peak }}$, the peak of the BBB disk emission (i.e., implement Equation (4)), calculate an accretion disk spectrum (as described in Section 4.1), calculate a non-thermal component spectrum (as described in Section 4.2), and sum these two components using a given weighting.

The output spectrum is a function of only three parameters, being $\log _{10}\left(E_{\text {peak }} / \mathrm{keV}\right), \Gamma$, and $p_{\mathrm{NT}}$, which is the proportion of the total flux over the range $0.01<E(\mathrm{keV})<20$, which is assigned to the non-thermal component, with $1-p_{\mathrm{NT}}$ being the proportion assigned to the BBB disk component.

The functions, which return the individual components and the full spectrum (sum of the two components), normalize the spectra so that the sum of the bin fluxes over the range of $0.01<E(\mathrm{keV})<20$ is equal to one.

\footnotetext{
4 Available at https://github.com/ADThomas-astro/oxaf and included in ASCL at http://ascl.net/1611.011.
}

\subsection{Validation}

The OXAF model was validated against the OPTXAGNF model by comparing OXAF output with OPTXAGNF output for many models across the parameter space. The BBB and non-thermal components were considered separately. The OPTXAGNF parameters used were chosen so that their various combinations did not give multiple identical BBB peak energies and hence multiple identical OXAF spectra. The chosen values were $\log _{10}\left(L / L_{\text {Edd }}\right)=-5.00,-3.75$, $-2.85, \quad-2.15, \quad-1.60, \quad-1.20, \quad-0.80, \quad-0.40, \quad 0.00$; $\log _{10}\left(M_{\mathrm{BH}} / M_{\odot}\right)=6.0,6.5,7.0,7.5,8.0,8.5,9.0 ; r_{\mathrm{cor}}=10$, $25,40,50,63,100 r_{g}$; and $\Gamma=1.4,1.7,2.0,2.3,2.6$, for a total of 1890 OPTXAGNF spectra. The range of parameters was chosen for completeness; not all of the tested parameter space necessarily corresponds to observed AGN.

Figures 8 and 9 in the Appendix demonstrate the performance of the OXAF model in reproducing the output spectra of OPTXAGNF. The figures show that over the relevant energy range, from the Lyman limit to above the energy at which iron may be fully ionized (0.01 to $\sim 20 \mathrm{keV})$, OXAF predictions are sufficiently close to OPTXAGNF predictions for our purposes (differences are far less than modeling uncertainties). The interquartile range displayed in each figure indicates that over the relevant energy range, OXAF predictions tend to be within $\sim 10 \%-20 \%$ of the OPTXAGNF value for the BBB component, and within $\sim 5 \%$ for the non-thermal component. The largest deviations tend to occur for energies that are probably too high to have a strong impact on photoionization model results $(1-10 \mathrm{keV})$ or are in an unlikely part of the parameter space (e.g., $r_{\text {cor }} \sim 60 r_{g}$ ). The most extreme discrepancies (i.e., 95th percentile and upward) for the BBB comparisons tend to be due to irrelevant cases, where only a small fraction of the total flux is in the relevant ionizing energy range, and hence the normalization over this range inflates a small section of the spectrum and magnifies proportional differences between the two models. For the non-thermal component the differences are due only to the small scatter in the fit given in Equation (7), i.e., the seed blackbody spectra for OXAF and OPTXAGNF have slightly different temperatures.

The OXAF spectra reproduce the OPTXAGNF models with sufficient accuracy for photoionization modeling of optical diagnostic emission lines. The differences between OXAF and OPTXAGNF are smaller than the uncertainties due to, for example, any of the following.

(a) The angular dependence of the accretion disk spectrum, including the effects of special relativistic beaming, gravitational beaming/light bending, and limb darkening (Laor \& Netzer 1989). Gravitational redshifts are an additional consideration.

(b) Uncertainties in fundamental aspects of the thin disk model, such as the $\alpha$-prescription, and aspects of the geometry of the accretion disk and corona including the variation with black hole spin (e.g., You et al. 2012). The luminosity range over which the model is applicable is another consideration, e.g., for luminosities over $\sim 0.3 L_{\text {Edd }}$ a "slim" disk is a more appropriate model.

(c) Radiative transfer effects; for example, the use of a color correction in the OPTXAGNF model neglects $\mathrm{H}$ and $\mathrm{He}$ edges (see Figure 1 in Done et al. 2012). 


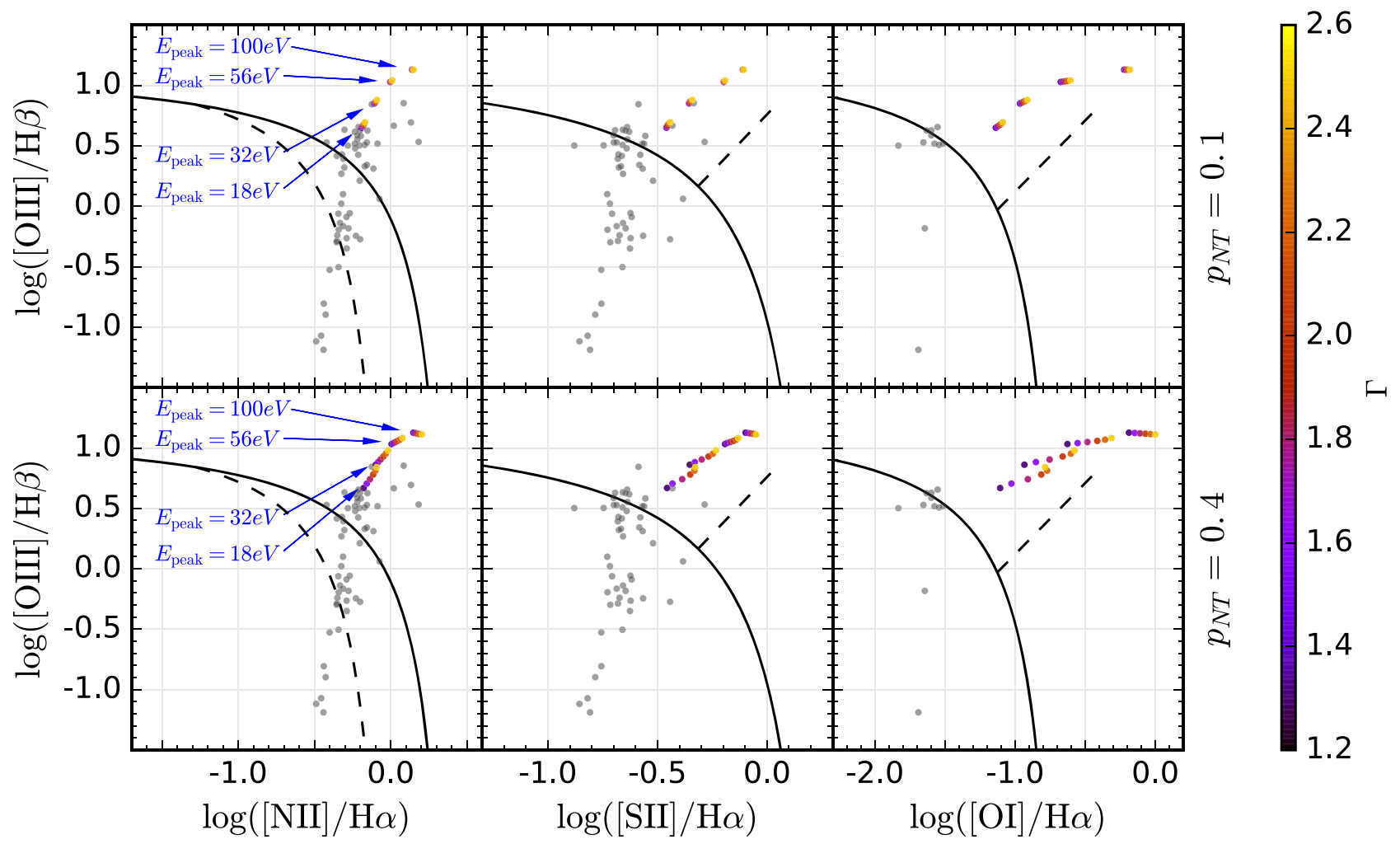

Figure 5. Optical diagnostic diagrams showing the effect of the OXAF parameters $E_{\text {peak }}, \Gamma$, and $p_{\mathrm{NT}}$ on MAPPINGS photoionization model predictions. Dividing lines are the same as in Figure 2. The top row of diagrams is for $p_{\mathrm{NT}}=0.1$ and the bottom row is for $p_{\mathrm{NT}}=0.4$. The gray points are observed line ratios for the galaxy NGC 1365 from the S7 galaxy survey (Dopita et al. 2015), with each point representing the line ratios for the narrowest Gaussian kinematic component in a single spatial pixel. See Section 5 in the text for more information and interpretation of the diagrams.

Two simple tests were performed to compare MAPPINGS 5.1 photoionization models based on OPTXAGNF and OXAF spectra. For the first test, the input OPTXAGNF spectrum was configured with $\left(L / L_{\mathrm{Edd}}, M_{\mathrm{BH}}, r_{\text {cor }}, \Gamma\right)=\left(0.2,10^{7} M_{\odot}, 15 r_{g}, 2.2\right)$, while the OXAF spectrum was configured with $\left(E_{\text {peak }}, \Gamma\right.$, $\left.p_{\mathrm{NT}}\right)=(68.3 \mathrm{eV}, 2.2,0.223)$, where $E_{\text {peak }}$ was calculated from Equation (4) and $p_{\mathrm{NT}}$ was calculated to match the proportion in the OPTXAGNF spectrum. The plane-parallel MAPPINGS models for this test were configured with a metallicity of $2 Z_{\odot}$, an ionization parameter of $U(H)=10^{-2}$, and a constant pressure of $P / k=10^{6} \mathrm{~K} \mathrm{~cm}^{-3}$. The resulting line fluxes were broadly consistent between the two models. Of 43 optical lines with predicted fluxes of more than $1 \%$ of $\mathrm{H} \beta$, all had a flux discrepancy of less than $10 \%$ of the flux predicted using the OPTXAGNF model. All but four line fluxes showed a difference of less than $5 \%$; these four lines from $\mathrm{N}$ I or O I had differences of $\sim 7 \%-8 \%$. The reason for this discrepancy is that these lines from low-ionization species are very sensitive to small differences in the shape of the hard photon spectrum since the emission is produced mostly in a partially ionized zone heated by Auger electrons.

For the second test, the input OPTXAGNF spectrum was configured with $\left(L / L_{\mathrm{Edd}}, M_{\mathrm{BH}}, r_{\mathrm{cor}}, \Gamma\right)=\left(0.3,10^{8} M_{\odot}, 20 r_{g}\right.$, $1.8)$, while the OXAF spectrum was configured with $\left(E_{\text {peak }}\right.$, $\left.\Gamma, p_{\mathrm{NT}}\right)=(19.9 \mathrm{eV}, 1.8,0.342)$. The MAPPINGS models used a metallicity of $3 Z_{\odot}$, an ionization parameter of $U(H)=10^{-2.5}$, and a constant pressure of $P / k=10^{6} \mathrm{~K} \mathrm{~cm}^{-3}$. With the somewhat softer ionizing spectrum and different nebular parameters, there were only 31 optical lines with predicted fluxes of more than $1 \%$ of $\mathrm{H} \beta$, and all of those lines had a flux discrepancy below $2 \%$, with 24 having a discrepancy under $1 \%$.

The accuracy of OXAF is more than sufficient for our purposes of photoionization modeling.

\section{THE EFFECT OF OXAF PARAMETERS ON PREDICTED EMISSION-LINE RATIOS}

A grid of MAPPINGS photoionization models was used to explore the effect of the OXAF parameters on emission-line ratios on the standard optical diagnostic diagrams. The grid was run over a range of photoionization model parameters and a range of all three OXAF parameters. The results are shown in Figure 5 for the dusty, plane-parallel MAPPINGS models configured with metallicity of $Z=1.5 Z_{\odot}$, ionization parameter of $U(H)=10^{-3}$, and constant pressure of $P / k=10^{7} \mathrm{~K} \mathrm{~cm}^{-3}$. Each model was iteratively re-run three times to ensure that the total pressure (including radiation pressure) was close to the input pressure, with this constraint applied where the model nebula was 50\% ionized. The iterations for each gridpoint culminated in a model that ended when the nebula was $99 \%$ neutral.

Also plotted in Figure 5 are gray points showing the measured spatially resolved line flux ratios for the galaxy NGC 1365 from the S7 galaxy survey (Dopita et al. 2015). Each point represents the line ratios for the narrowest Gaussian 
kinematic component in the spectrum of a $1^{\prime \prime} \times 1^{\prime \prime}$ spatial pixel (the seeing FWHM was $11^{\prime \prime} 1$ ). An S/N cut of 10 was applied to the linear flux ratios. In the leftmost two columns of diagrams, these points form a "mixing sequence" from line ratios associated with excitation purely due to star formation (at the bottom) to excitation purely by AGN radiation (at the top).

The predicted line ratios for our fiducial MAPPINGS models approximately coincide with some of the measured "pureAGN" line ratios, especially in the leftmost column. Predictions of the weaker [O I] $\lambda 6300$ line, however, are inconsistent with the observations. This line is produced predominantly in the partially ionized zone that arises due to the hard EUV and X-ray photons. The temperature and size of this region and thus the strength of the [O I] line are sensitive to the hard ionizing spectrum. In addition, the fractional ionization state of oxygen is strongly dependent upon charge exchange reactions and, as a result, this line is very difficult to predict accurately. The $[\mathrm{S} \mathrm{II}] \lambda \lambda 6716,6731$ doublet can also arise from this partially ionized region and is also somewhat inconsistent with the observations, albeit to a lesser degree. The overpredicted fluxes from these species could to some extent be due to the NLR clouds being mass-bounded as opposed to ionization-bounded (as in the models), i.e., more of the high-energy photons escape and the partially ionized zones are shorter for the observed ensemble of NLR clouds in NGC 1365 compared to the models.

\subsection{Effect of the Individual OXAF Parameters}

In this section, we discuss Figure 5 and interpret the effect of each OXAF parameter on the model predictions.

\subsubsection{Energy of the Peak of the Disk Emission $E_{\text {Peak }}$}

The logarithmically spaced values of $E_{\text {peak }}$ in Figure 5 show that increasing the energy of the peak of the disk emission, and thereby increasing the energy of the ionizing photons, increases both the relative ionization state and the temperature of the nebula. The sensitivity of optical diagnostic line ratios to $E_{\text {peak }}$ is comparable to the sensitivity to the ionization parameter.

\subsubsection{Proportion of Flux in Non-thermal Component, $p_{N T}$}

We first consider the expected range of values of $p_{\mathrm{NT}}$. Jin et al. (2012a) study a sample of 51 unobscured Seyfert 1 galaxies and fit the full three-component OPTXAGNF model to the observed SEDs. The proportion of the bolometric luminosity emitted in the power-law component in the bestfit model is less than 0.05 for $\sim 20 \%$ of the sample, less than 0.2 for $\sim 50 \%$ of the sample, and less than 0.4 for $\sim 80 \%$ of the sample. Although the soft excess is a significant component (allocated more flux than the power-law component for $\sim 70 \%$ of the sample), it is effectively an extension of accretion disk emission to higher energies, so the proportion of flux in the power-law tail is comparable to the $p_{\mathrm{NT}}$ OXAF parameter. Hence, we take $p_{\mathrm{NT}} \sim 0.1-0.4$ as an appropriate range for typical ionizing AGN spectra.

The values $p_{\mathrm{NT}}=0.2,0.7$ were included in the model grid but are not shown in Figure 5. The diagnostic line-ratio predictions for $p_{\mathrm{NT}}=0.2$ were intermediate between those of $p_{\mathrm{NT}}=0.1$ and $p_{\mathrm{NT}}=0.4$ and were similar to those of $p_{\mathrm{NT}}=0.1$. The value $p_{\mathrm{NT}}=0.7$ was very high, with only a few extreme objects in the Jin et al. (2012a) sample having

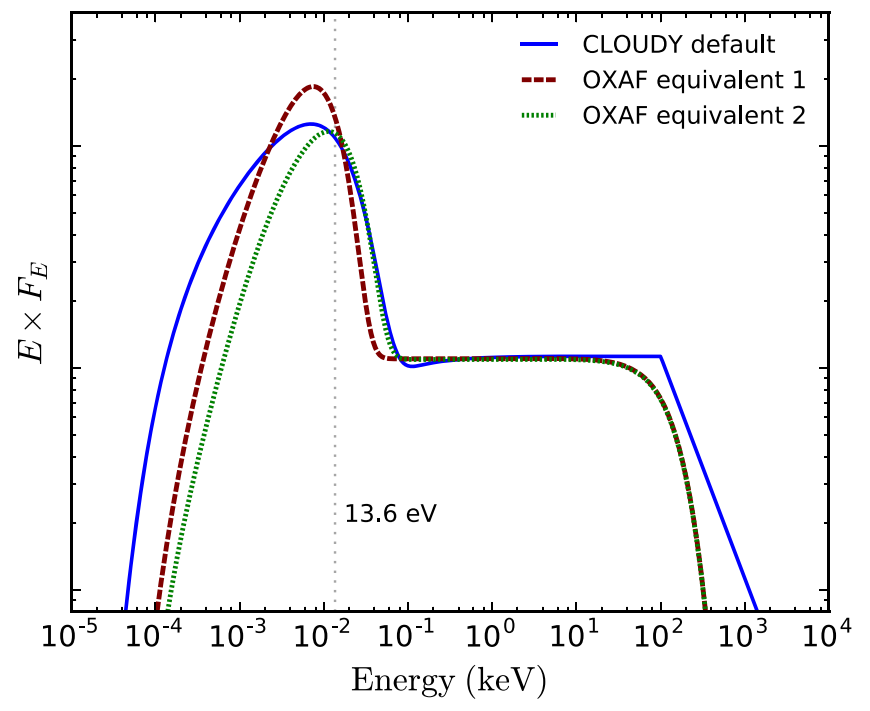

Figure 6. Comparison between a default CLOUDY ionizing AGN spectrum and two OXAF spectra chosen to be "equivalent" in differing ways. The parameters used for the spectra are listed in Section 5.2.

such power-law-dominated SEDs. Hence the figure requires only the values $p_{\mathrm{NT}}=0.1,0.4$ to indicate the effect of $p_{\mathrm{NT}}$ and show the approximate magnitude of strong line-ratio variations due to $p_{\mathrm{NT}}$ variations that may occur between Seyfert galaxies.

Increasing the value of $p_{\mathrm{NT}}$ increases the length of the trailing partially ionized zone in the photoionization models. For $E_{\text {peak }}=32 \mathrm{eV}, \Gamma=1.8, \log U=-2.0$ and $Z=1.5 Z_{\odot}$, variation of $p_{\mathrm{NT}}$ from 0.1 to 0.7 increased the length of the modeled cloud by a factor of $\sim 4$ to $1.5 \mathrm{pc}$. Extending the partially ionized zone increases the $[\mathrm{S} \mathrm{II}] / \mathrm{H} \alpha$ and $[\mathrm{O} \mathrm{I}] / \mathrm{H} \alpha$ line ratios, as the figure shows for $p_{\mathrm{NT}}=0.1 \rightarrow 0.4$.

\subsubsection{Photon Index of the Power-law Tail $\Gamma$}

For values of $p_{\mathrm{NT}} \lesssim 0.2$, the predicted emission-line ratios do not show strong sensitivity to $\Gamma$. Figure 5 shows how as $\Gamma$ increases, [S II] and [O I] line emission is enhanced relative to $\mathrm{H} \alpha$. Increasing $\Gamma$ allocates more of the non-thermal energy to relatively soft X-ray photons that are more easily absorbed by the nebula, causing more emission in the partially ionized zone. Hence, $\Gamma$ has a similar effect to $p_{\mathrm{NT}}$. As $p_{\mathrm{NT}}$ increases, the sensitivity to $\Gamma$ increases due to the greater relative effect of $\mathrm{X}$-ray power-law photons on the nebula ionization structure.

\subsection{Comparison with Default CLOUDY AGN Spectrum}

In this section, we compare OXAF spectra with the default spectrum produced by the AGN spectral model supplied with the CLOUDY (Ferland et al. 2013) photoionization code. We also compare the predicted optical diagnostic emission-line ratios produced by using these ionizing spectra in MAPPINGS photoionization models.

The CLOUDY AGN spectral model uses an analytic formula consisting of exponential and power-law factors and terms. The default CLOUDY AGN model was configured with the following default values: a BBB temperature of $T=1.5 \times 10^{5} \mathrm{~K}$, an $\mathrm{X}$-ray to UV ratio of $\alpha_{\mathrm{ox}}=-1.4$, a low-energy BBB slope of 


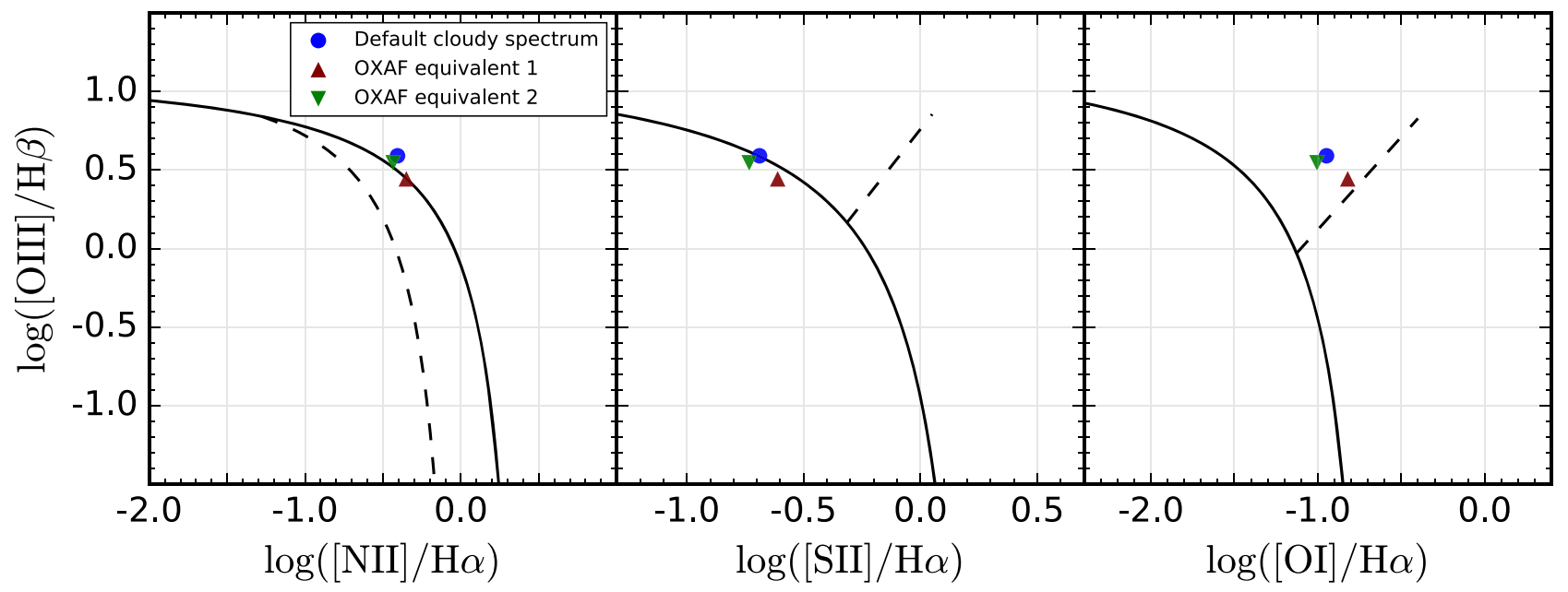

Figure 7. Optical diagnostic diagram showing predicted emission-line flux ratios from MAPPINGS photoionization models using the three different ionizing spectra shown in Figure 6. The dividing lines are the same as in Figure 2. The three ionizing spectra are the default CLOUDY AGN spectrum and two OXAF spectra that are "equivalent" in differing ways to the CLOUDY spectrum; colors in this figure correspond to the colors of these three spectra in Figure 6. The plane-parallel dusty MAPPINGS models were all configured with a metallicity of $3 Z_{\odot}$, an ionization parameter of $\log U=-2.0$, and a constant pressure of $P / k=10^{6}$.

$\alpha_{\mathrm{uv}}=-0.5$, and an X-ray power-law index of $\alpha_{x}=-1$ (corresponding to $\Gamma=2$ ).

Figure 6 shows the CLOUDY default SED, along with two "equivalent" OXAF spectra. The first OXAF spectrum has $\left(E_{\text {peak }}, \Gamma, p_{\mathrm{NT}}\right)=(6.9 \mathrm{eV}, 2.0,0.46)$, chosen to match the BBB peak energy and the "height" of the power-law tail, and has the same normalization as the normalized CLOUDY default SED. The second "equivalent" OXAF spectrum has $\left(E_{\text {peak }}\right.$, $\left.\Gamma, p_{\mathrm{NT}}\right)=(9.9 \mathrm{eV}, 2.0,0.414)$ to match the shape and position of the high-energy slope of the BBB and match the "height" of the power-law tail. This second OXAF spectrum is shown with a different normalization, with only $73 \%$ of the energy flux of the CLOUDY spectrum, to show how it follows the shape of the CLOUDY spectrum in the important region associated with $\mathrm{H}$-ionizing FUV photons.

Clear differences between the spectral models are apparent in Figure 6. The CLOUDY model uses a single blackbody curve combined with a power law to produce the $\mathrm{BBB}$, resulting in a BBB much wider than the OXAF BBB. The physical model used to calculate the power-law tail in OXAF produces a powerlaw cutoff that is much steeper than the simple piecewise $\nu^{-2}$ cutoff in the CLOUDY model.

Results of MAPPINGS photoionization model runs using the three spectra in Figure 6 are presented in Figure 7. The planeparallel dusty MAPPINGS models were all configured with a metallicity of $3 Z_{\odot}$, an ionization parameter of $\log U=-2.0$, and a constant pressure of $P / k=10^{6}$. All three models produced similar optical diagnostic line ratios. The second OXAF model resulted in line ratios closer to those due to the CLOUDY default spectrum, which confirms that the most important part of the spectrum for ionization modeling is the region immediately on the high-energy side of the $\mathrm{H}$ ionization threshold, where these spectra were very similar by design. The first OXAF model results in relatively lower $[\mathrm{O} \mathrm{III}] / \mathrm{H} \beta$, which is presumably a consequence of the lower proportion of hydrogen-ionizing EUV photons.

The results show that OXAF spectra may produce predicted diagnostic line ratios that are very similar to those resulting from analytic ionizing spectra not based on physical models, provided the spectral shapes are sufficiently well-matched in the most important part of the spectrum, the hydrogenionizing EUV.

\section{CONCLUSIONS}

We present a model of AGN continuum emission, OXAF, designed for use in photoionization modeling and featuring spectral shapes based on physical models. The OXAF model removes degeneracies between AGN parameters in terms of their effect on the ionizing spectral shape; in particular, in the thin disk accretion model the black hole mass and AGN luminosity are entirely degenerate with respect to their impact on the spectral shape. We base OXAF on the OPTXAGNF model (Done et al. 2012), and explain and remove some additional spectral-shape degeneracies between AGN parameters when we reparametrize OPTXAGNF.

We show that the soft X-ray excess is not important to photoionization modeling of the standard optical strong diagnostic lines, so we do not include it in the OXAF model. However, the soft excess must be important in modeling forbidden high-ionization lines.

The model OXAF contains only three parameters - the energy of the peak of the accretion disk emission $E_{\text {peak }}$, the photon power-law index for the non-thermal Comptonized component $\Gamma$, and the proportion of the total flux, which goes to the nonthermal component $p_{\mathrm{NT}}$. These parameters intuitively describe the physical shape of the produced spectrum.

We show that predicted ratios of strong lines on standard optical diagnostic diagrams are sensitive to all three OXAF parameters. The parameter $E_{\text {peak }}$ directly affects the degree of ionization of the MAPPINGS model nebulae. The parameters $\Gamma$ and $p_{\mathrm{NT}}$ are similar in their effects in that they change the length of the partially ionized zone. Predicted line ratios are more sensitive to $\Gamma$ as $p_{\mathrm{NT}}$ is increased. Measured strong emission-line ratios for the Seyfert galaxy NGC 1365 are approximately consistent with the predictions of some fiducial photoionization models using input OXAF ionizing spectra. 
Users of OXAF may further explore the effects of changing the shape of the ionizing spectrum on predicted emission-line spectra.

B.G. gratefully acknowledges the support of the Australian Research Council as the recipient of a Future Fellowship (FT140101202). L.K. and M.D. acknowledge support from ARC discovery project \#DP160103631. C.J. acknowledges the support by the Bundesministerium für Wirtschaft und Technologie/Deutsches Zentrum für Luft- und Raumfahrt
(BMWI/DLR, FKZ 50 OR 1604) and the Max Planck Society. We are grateful to both Chris Done, who received an early draft of this paper, and the anonymous referee for helpful comments.

\section{APPENDIX}

Plots comparing OXAF with OPTXAGNF are presented in Figures 8 and 9 .

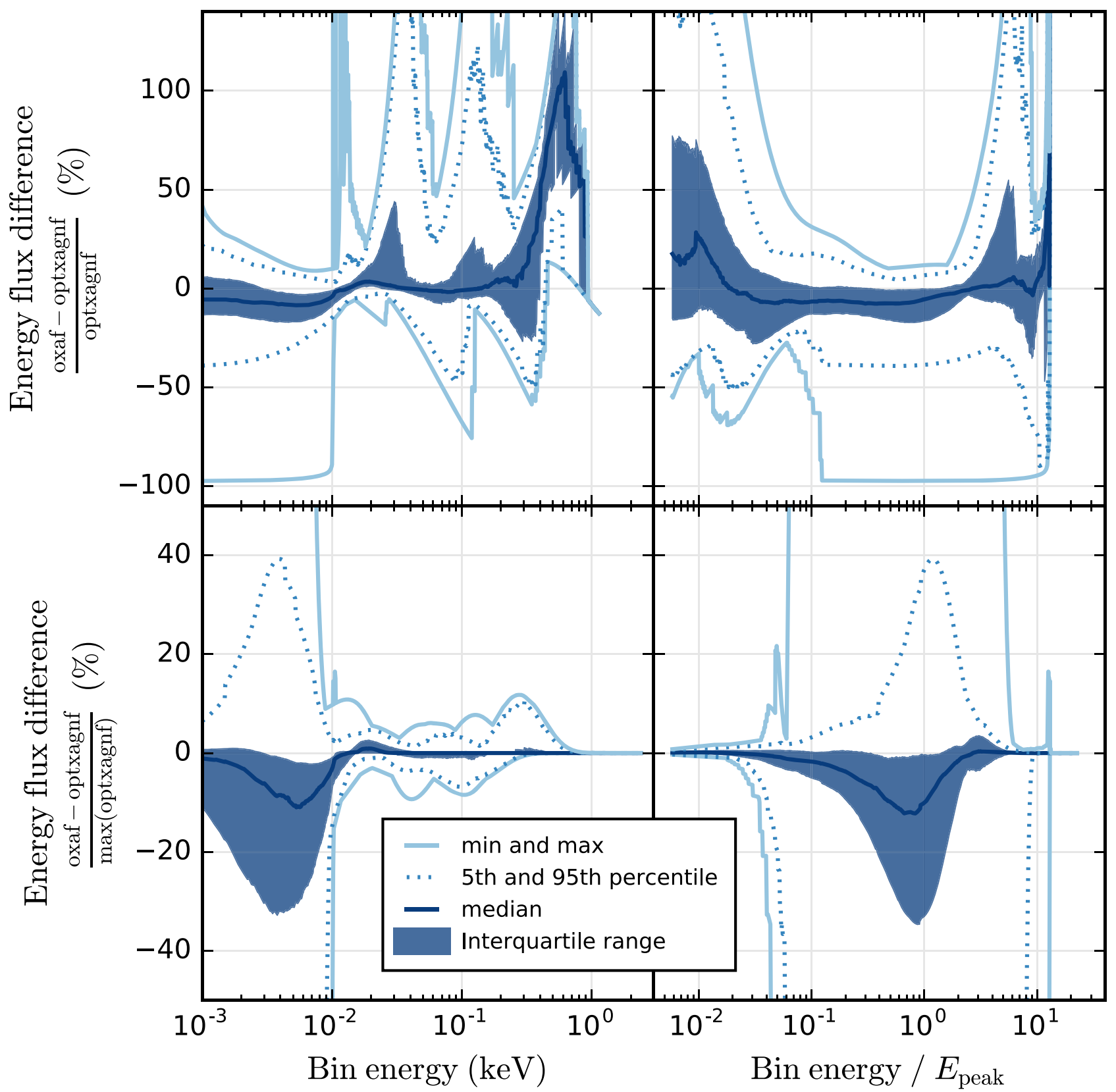

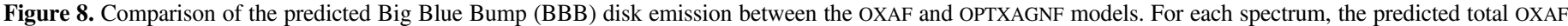

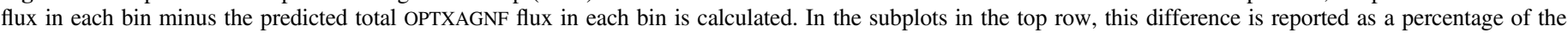

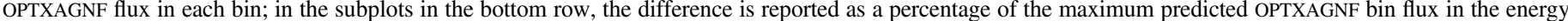

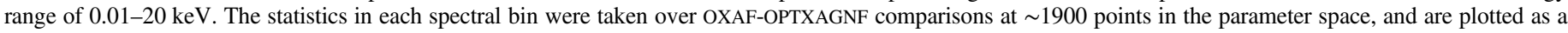

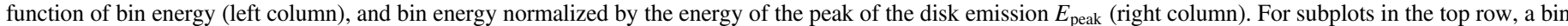

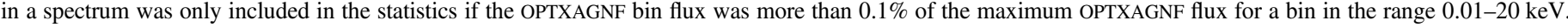
The accuracy of OXAF in reproducing the OPTXAGNF spectra is more than adequate over the relevant energy range of $0.01-\sim 20 \mathrm{keV}$. 


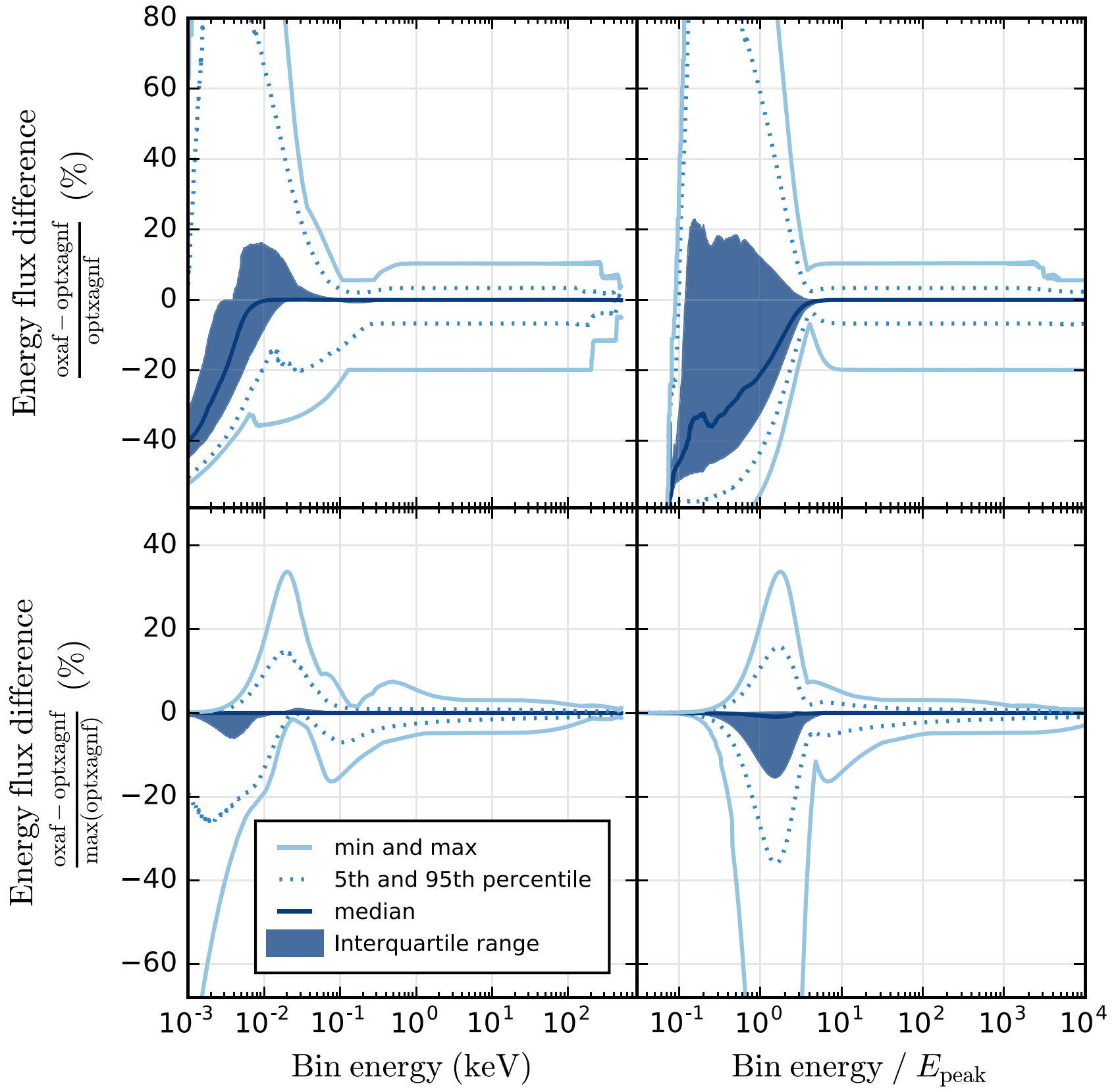

Figure 9. Comparison of the predicted hard non-thermal emission between the OXAF and OPTXAGNF models. For each spectrum, we calculate the predicted total OXAF flux in each bin minus the predicted total OPTXAGNF flux in each bin. In the subplots in the top row, this difference is reported as a percentage of the OPTXAGNF flux in each bin; in the subplots in the bottom row, the difference is reported as a percentage of the maximum predicted OPTXAGNF bin flux in the energy range 0.01-20 keV. The statistics in each spectral bin were taken over OXAF-OPTXAGNF comparisons at $\sim 1900$ points in the parameter space, and are plotted as a function of bin energy (left column), and bin energy normalized by the energy of the peak of the disk emission $E_{\text {peak }}$ (right column). For subplots in the top row, a bin in a spectrum was only included in the statistics if the OPTXAGNF bin flux was more than $0.1 \%$ of the maximum OPTXAGNF flux for a bin in the range $0.01-20 \mathrm{keV}$. The accuracy of OXAF in reproducing the OPTXAGNF spectra is more than adequate over the relevant energy range of $0.01-\sim 20 \mathrm{keV}$.

\section{REFERENCES}

Allen, M. G., Dopita, M. A., \& Tsvetanov, Z. I. 1998, ApJ, 493, 571

Antonucci, R. 1993, ARA\&A, 31, 473

Baldwin, J. A., Phillips, M. M., \& Terlevich, R. 1981, PASP, 93, 5

Bianchi, S., Guainazzi, M., \& Chiaberge, M. 2006, A\&A, 448, 499

Bland-Hawthorn, J., Maloney, P. R., Sutherland, R. S., \& Madsen, G. J. 2013, ApJ, 778, 58

Collins, N. R., Kraemer, S. B., Crenshaw, D. M., Bruhweiler, F. C., \& Meléndez, M. 2009, ApJ, 694, 765

Contini, M., \& Viegas, S. M. 2001, ApJS, 132, 211

Czerny, B., You, B., Kurcz, A., et al. 2016, A\&A, 594, A102

Davies, R. L., Dopita, M. A., Kewley, L., et al. 2016, ApJ, 824, 50
Davis, S. W., Done, C., \& Blaes, O. M. 2006, ApJ, 647, 525

De Marco, B., Ponti, G., Cappi, M., et al. 2013, MNRAS, 431, 2441

Done, C., Davis, S. W., Jin, C., Blaes, O., \& Ward, M. 2012, MNRAS, 420, 1848

Dopita, M. A., Groves, B. A., Sutherland, R. S., Binette, L., \& Cecil, G. 2002, ApJ, 572, 753

Dopita, M. A., Scharwächter, J., Shastri, P., et al. 2014, A\&A, 566, A41

Dopita, M. A., Shastri, P., Davies, R. L., et al. 2015, ApJS, 217, 12

Evans, I., Koratkar, A., Allen, M., Dopita, M., \& Tsvetanov, Z. 1999, ApJ, 521, 531

Fabian, A. C. 2016, AN, 337, 375

Ferland, G. J., Porter, R. L., van Hoof, P. A. M., et al. 2013, RMxAA, 49, 137

Gierliński, M., \& Done, C. 2004, MNRAS, 349, L7 
Groves, B. A., Dopita, M. A., \& Sutherland, R. S. 2004, ApJS, 153, 9

Groves, B. A., Heckman, T. M., \& Kauffmann, G. 2006, MNRAS, 371, 1559

Jin, C., Ward, M., \& Done, C. 2012, MNRAS, 422, 3268

Jin, C., Ward, M., \& Done, C. 2012, MNRAS, 425, 907

Jin, C., Ward, M., Done, C., \& Gelbord, J. 2012, MNRAS, 420, 1825

Kauffmann, G., Heckman, T. M., Tremonti, C., et al. 2003, MNRAS, 346, 1055

Kewley, L. J., Dopita, M. A., Sutherland, R. S., Heisler, C. A., \& Trevena, J. 2001, ApJ, 556, 121

Kewley, L. J., Groves, B., Kauffmann, G., \& Heckman, T. 2006, MNRAS, 372,961

Laor, A., \& Netzer, H. 1989, MNRAS, 238, 897

Lightman, A. P., \& Zdziarski, A. A. 1987, ApJ, 319, 643

Molina, M., Bassani, L., Malizia, A., et al. 2013, MNRAS, 433, 1687

Murayama, T., \& Taniguchi, Y. 1998, ApJL, 503, L115
Novikov, I. D., \& Thorne, K. S. 1973, in Black Holes (Les Astres Occlus), ed. C. DeWitt \& B. S. DeWitt (Philadelphia, PA: Gordon \& Breach Science), 343

Page, D. N., \& Thorne, K. S. 1974, ApJ, 191, 499

Pal, M., Dewangan, G. C., Misra, R., \& Pawar, P. K. 2016, MNRAS, 457, 875

Reynolds, C. S. 2014, SSRv, 183, 277

Shakura, N. I., \& Sunyaev, R. A. 1973, A\&A, 24, 337

Veilleux, S., \& Osterbrock, D. E. 1987, ApJS, 63, 295

Viegas-Aldrovandi, S. M., \& Contini, M. 1989, A\&A, 215, 253

Vika, M., Driver, S. P., Graham, A. W., \& Liske, J. 2009, MNRAS, 400, 1451

Walton, D. J., Nardini, E., Fabian, A. C., Gallo, L. C., \& Reis, R. C. 2013, MNRAS, 428, 2901

You, B., Cao, X., \& Yuan, Y.-F. 2012, ApJ, 761, 109

Zdziarski, A. A., Johnson, W. N., \& Magdziarz, P. 1996, MNRAS, 283, 193

Życki, P. T., Done, C., \& Smith, D. A. 1999, MNRAS, 309, 561 